Article

\title{
Single Sodium Pyruvate Ingestion Modifies Blood Acid-Base Status and Post-Exercise Lactate Concentration in Humans
}

\author{
Robert A. Olek $^{1}{ }^{1}$, Sylwester Kujach ${ }^{2}$, Damian Wnuk ${ }^{3}$ and Radoslaw Laskowski ${ }^{2}$
}

1 Biochemistry Department, Gdansk University of Physical Education and Sport, Gorskiego 1, 80-336 Gdansk, Poland

2 Physiology Department, Gdansk University of Physical Education and Sport, Gorskiego 1, 80-336 Gdansk, Poland; E-Mails: sylwek-kujach@o2.pl (S.K.); lasradek@awf.gda.pl (R.L.)

3 Department of Physiotherapy, Medical University of Gdansk, Debinki 7, 80-211 Gdansk, Poland; E-Mail: damianwnuk@gumed.edu.pl

* Author to whom correspondence should be addressed; E-Mail: robol@awf.gda.pl;

Tel.: +48-58-5547-214; Fax: +48-58-552-2911.

Received: 17 February 2014; in revised form: 30 April 2014 / Accepted: 6 May 2014 /

Published: 16 May 2014

\begin{abstract}
This study examined the effect of a single sodium pyruvate ingestion on a blood acid-base status and exercise metabolism markers. Nine active, but non-specifically trained, male subjects participated in the double-blind, placebo-controlled, crossover study. One hour prior to the exercise, subjects ingested either $0.1 \mathrm{~g} \cdot \mathrm{kg}^{-1}$ of body mass of a sodium pyruvate or placebo. The capillary blood samples were obtained at rest, $60 \mathrm{~min}$ after ingestion, and then three and $15 \mathrm{~min}$ after completing the workout protocol to analyze acid-base status and lactate, pyruvate, alanine, glucose concentrations. The pulmonary gas exchange, minute ventilation and the heart rate were measured during the exercise at a constant power output, corresponding to $\sim 90 \% \dot{\mathrm{V}} \mathrm{O}_{2} \max$. The blood $\mathrm{pH}$, bicarbonate and the base excess were significantly higher after sodium pyruvate ingestion than in the placebo trial. The blood lactate concentration was not different after the ingestion, but the post-exercise was significantly higher in the pyruvate trial $(12.9 \pm 0.9 \mathrm{mM})$ than in the placebo trial $(10.6 \pm 0.3 \mathrm{mM}, p<0.05)$ and remained elevated (nonsignificant) after $15 \mathrm{~min}$ of recovery. The blood pyruvate, alanine and glucose concentrations, as well as the overall pulmonary gas exchange during the exercise were not affected by the pyruvate ingestion. In conclusion, the sodium pyruvate ingestion one hour before workout modified the blood acid-base status and the lactate production during the exercise.
\end{abstract}


Keywords: sodium pyruvate; exercise; blood acid-base status; lactate; alanine

\section{Introduction}

Pyruvate (PYR) participates in several various reactions and plays a key role in energy metabolism. It is produced from glucose (GLU) in the cytoplasm of skeletal muscle cells by the glycolytic pathway. During this process, $\mathrm{NAD}^{+}$is reduced to $\mathrm{NADH}$ in the reaction catalyzed by glyceraldehyde-3-phosphate dehydrogenase (G3PDH). The slow rate of glycolysis in the low intensity exercise allows all produced PYR and reducing equivalents to be transferred to mitochondria. Inside the mitochondria, PYR is decarboxylated by the pyruvate dehydrogenase complex (PDC) to acetyl-CoA, which enters the tricarboxylic acid (TCA) cycle. PDC and TCA cycle dehydrogenases generate reducing equivalents used for ATP production in the oxidative phosphorylation process performed in the electron transport chain. On the other hand, a high energy demand during an intensive exercise raises the rate of PYR production. PYR is reduced to lactate (LA) by the cytoplasmic NADH in the reaction catalyzed by the lactate dehydrogenase (LDH). This mechanism enables PYR to increase the cytosolic ATP resynthesis rate (for a review, see [1]). The exercise intensity elevation also causes a higher release of alanine (ALA) from the skeletal muscles [2]. ALA is produced by PYR transamination. In the same reaction, $\alpha$-ketoglutarate, an intermediate of TCA cycle, is formed.

It has been shown that PYR diminishes cellular and organ dysfunction during hypoxic conditions [3-7]. The increased LA production [4] and the direct NADH measurement [3] indicate that the presence of exogenous PYR improves the cytosolic NADH reoxidation. Therefore, the blockage of G3PDH by a high NADH/NAD ${ }^{+}$ratio is moderated, and ATP production by anaerobic glycolysis may be stimulated [8]. Moreover, Robergs et al. [9] suggested that PYR reduction to LA consumes $\mathrm{H}^{+}$; therefore, PYR may act as a buffer. The buffering properties of PYR have been directly shown, while it has been used in a perfusing solution of the isolated organs $[4,8]$ or during intravenous infusion $[5-7,10]$. However, in all these studies, PYR has been used in supraphysiological concentrations [4-8,10].

Despite the importance of PYR in energy metabolism, no ergogenic effects have been shown after a prolonged supplementation [11,12]. PYR intake for seven days failed to enhance the performance during an intense aerobic exercise in well-trained individuals [11]. Furthermore, PYR consumption for 14 consecutive days does not improve exercise capacity, as measured by a critical power cycle ergometer test [12]. Moreover, Morrison et al. [11] indicated that acute oral intake of calcium PYR $(\mathrm{CaP})$, even at a dose of $25 \mathrm{~g}$, did not modify the PYR concentration in the whole blood or in the plasma. However, we have recently found that single sodium PYR (NaP) and CaP ingestion increased blood $\mathrm{pH}$, but $\mathrm{NaP}$ was more effective in the blood bicarbonate level modification [13] (unpublished). Therefore, the purpose of this study is to examine the effect of a single NaP ingestion on blood acid-base status and the exercise metabolism markers.

Since $0.1 \mathrm{~g}$ of sodium bicarbonate per $\mathrm{kg}$ of body mass induces metabolic alkalosis $60 \mathrm{~min}$ following ingestion $[14,15]$, we hypothesized that a similar $\mathrm{NaP}$ treatment before commencing the high intensity physical exertion may change the exercise metabolism. 


\section{Experimental Section}

\subsection{Subjects}

Nine active, but non-specifically trained, male subjects (mean \pm SEM: $23 \pm 1$ year old, $1.75 \pm 0.02 \mathrm{~m}$ height, $72 \pm 2 \mathrm{~kg}$ body mass) participated in the double-blind, placebo-controlled, crossover study. The study was approved by the Local Ethics Committee, and all subjects signed an informed consent before the start of the study. The subjects were asked to refrain from any physical activity or alcohol consumption for at least $24 \mathrm{~h}$ and caffeine for at least $12 \mathrm{~h}$ prior to testing.

\subsection{Procedures}

To determine $\dot{\mathrm{V}} \mathrm{O}_{2} \max$, participants performed a graded cycle ergometry test on an electromagnetically-braked, cycle ergometer (ER 900 Jaeger, Viasys Healthcare GmbH, Hoechberg, Germany). The height of the ergometer seat was individually adjusted, and the participants were allowed a 5-min warm-up period at an intensity of $1.5 \mathrm{~W} \cdot \mathrm{kg}^{-1}$ with a pedaling cadence of $60 \mathrm{rpm}$. After the warm-up period, the work rate was increased by $25 \mathrm{~W} \cdot \mathrm{min}^{-1}$ until volitional exhaustion [16]. Breath by breath pulmonary gas exchange was measured by Oxycon-Pro analyzer (Viasys Healthcare $\mathrm{GmbH}$, Hoechberg, Germany), and the $\mathrm{O}_{2}$ and $\mathrm{CO}_{2}$ analyzers were calibrated prior to each test using standard gases of known concentrations in accordance with manufacturer guidelines. The heart rates were monitored continuously by telemetry (S-625, Polar Electro-Oy, Kempele, Finland) during each test session and the first $5 \mathrm{~min}$ of passive recovery in a seated position. After the $\dot{\mathrm{V}} \mathrm{O}_{2} \mathrm{max}$ test, subjects visited the laboratory for a practice ride, which familiarized the subjects with the experiment protocol and confirmed the power output ( $\left.\sim 90 \% \dot{\mathrm{V}} \mathrm{O}_{2} \mathrm{max}\right)$.

\subsection{Measurements}

On separate days, the subjects reported to the laboratory in the morning, then rested for $30 \mathrm{~min}$ and then ingested placebo or $\mathrm{NaP}$ in a random order. In the previous studies the subjects consumed PYR in the amount of $\sim 0.07-0.08 \mathrm{~g} \cdot \mathrm{kg}^{-1}$ body mass $[17,18]$; therefore, the subjects in our study ingested NaP in a single dose of $0.1 \mathrm{~g} \cdot \mathrm{kg}^{-1}$ body mass (which is $\sim 0.08 \mathrm{~g}$ of PYR per $\mathrm{kg}$ body mass). Moreover, it has been reported that the same dose of sodium bicarbonate induces metabolic alkalosis [14,15]. An hour following the ingestion, the subjects performed the physical exertion. The exercise protocol consisted of $2 \mathrm{~min}$ at a power output of $50 \mathrm{~W}$ and then for $6 \mathrm{~min}$ at a constant power output, corresponding to $\sim 90 \% \dot{\mathrm{V}} \mathrm{O}_{2}$ max. The respiratory gas analysis and the volume measurements were performed breath by breath with a face-mask connected to the analyzer. The breath-by-breath pulmonary $\dot{\mathrm{V}} \mathrm{O}_{2}$ was measured continuously throughout the exercise by an Oxycon-Pro gas analyzing system (Viasys Healthcare $\mathrm{GmbH}$, Hoechberg, Germany). The data were first manually filtered to remove outlying breaths, defined as breaths deviating by more than three standard deviations from the preceding five breaths. The data were subsequently interpolated to provide second-by-second values, and then, the slow component amplitude was estimated by calculating the difference between the mean $\dot{\mathrm{V}} \mathrm{O}_{2}$ during the last $60 \mathrm{~s}$ of the exercise and the mean $\dot{\mathrm{V}} \mathrm{O}_{2}$ during the 60 -s period on third minute of exercise [19]. 


\subsection{Blood Analysis}

At rest, $60 \mathrm{~min}$ after $\mathrm{NaP}$ or the placebo ingestion, and then 3 and $15 \mathrm{~min}$ after completing the exercise protocol, the blood samples were taken from the fingertips into heparinized capillary tubes (Siemens Healthcare Diagnostics Inc., Tarrytown, NY, USA) and were immediately analyzed for blood gases, pH, hemoglobin ( $\mathrm{Hb}$ ) and electrolytes using a Rapidpoint 400/405 (Siemens Healthcare Diagnostics Inc., Tarrytown, NY, USA). In addition, the bicarbonate $\left(\mathrm{HCO}_{3}{ }^{-}\right)$was calculated from $\mathrm{PCO}_{2}$ and $\mathrm{pH}$ values according to the Henderson-Hasselbalch equation, and the base excess (BE) was calculated according to the following equation [20]:

$$
\mathrm{BE}=(1-0.014 \times[\mathrm{Hb}]) \times\left(\left[\mathrm{HCO}_{3}{ }^{-}\right]-24.8+(1.43 \times[\mathrm{Hb}]+7.7) \times(\mathrm{pH}-7.4)\right)
$$

The blood samples for GLU, LA, PYR and ALA analysis were immediately deproteinized by the addition of ice cold $0.4 \mathrm{M}$ perchloric acid. After being thoroughly mixed, the samples were centrifuged at $12,000 \times g$ for $10 \mathrm{~min}$ and stored for later analysis. GLU concentration was quantified using a standard test kit (Randox Laboratories Ltd., Crumlin, UK) based on the glucose oxidase method (GL2623). Assays were performed on Super Aquarius CE9200 (Cecil Instruments Ltd., Cambridge, UK). LA, PYR and ALA concentrations were determined fluorometrically by the method of Maughan [21], which is based on the enzymatic reactions of NAD ${ }^{+}$reduction (LA, ALA) or NADH oxidation (PYR). The fluorescence intensity was measured at an excitation maximum of $340 \mathrm{~nm}$ and an emission maximum of $460 \mathrm{~nm}$ on an EnSpire Multimode Plate Reader (Perkin Elmer Inc., Waltham, MA, USA). The known concentrations of the compounds were used to construct a standard curve. All the reagents were obtained from Sigma-Aldrich Co. (Schnelldorf, Germany).

\subsection{Statistics}

The statistical analyses were performed using STATISTICA 9.0 (Statsoft Inc., Tulsa, OK, USA) software, with the level of significance set at $p<0.05$. A repeated-measures ANOVA across treatments (placebo and $\mathrm{NaP}$ ) and time (rest, $60 \mathrm{~min}$ after ingestion, $3 \mathrm{~min}$ after the exercise and $15 \mathrm{~min}$ of recovery) were used to assess the differences in the blood acid-base status and metabolites. Furthermore, due to the small number of subjects, we also calculated the effect size (partial $\eta^{2}$ ). It is a measure of effect size for use in ANOVA, ranging between 0 and 1. Using Cohen's rule of thumb, as well as the conversion table for $\eta^{2}$, the interpretations of the partial $\eta^{2}$ value are unequivocal. However, the most restricted interpretation method assigns values to the effect size as follows: 0.1 , a small effect; 0.3 , a medium effect; and 0.5 , a large effect. The pulmonary gas exchange parameters were analyzed applying a Student's $t$-test.

\section{Results}

The ingestion of $\mathrm{NaP}$ affected the blood acid-base status (Figure 1). The $\mathrm{pH}$ was significantly higher in the NaP trial than in the placebo trial $\left(p=0.015\right.$, partial $\eta^{2}=0.54$; Figure 1A). Similarly, the blood bicarbonate concentration (Figure 1B) and BE (Figure 1C) increased after NaP ingestion and remained above the placebo conditions. The main treatment effects for $\mathrm{HCO}_{3}{ }^{-}$and $\mathrm{BE}$ were $\left(p=0.005\right.$, partial $\eta^{2}=0.65 ;$ and $p=0.003$, partial $\left.\eta^{2}=0.69\right)$, respectively. 
Figure 1. Blood $\mathrm{pH}(\mathbf{A}), \mathrm{HCO}_{3}(\mathbf{B})$, and base excess (BE) (C) responses measured before placebo $(\bullet)$ and $\mathrm{NaP}(\circ)$ ingestion (Pre-Ing.), 60 min after ingestion (Post-Ing.), 3 min after completion of exercise (Post-Ex.) and $15 \mathrm{~min}$ of recovery (15-Post). Values are the means \pm SEM.
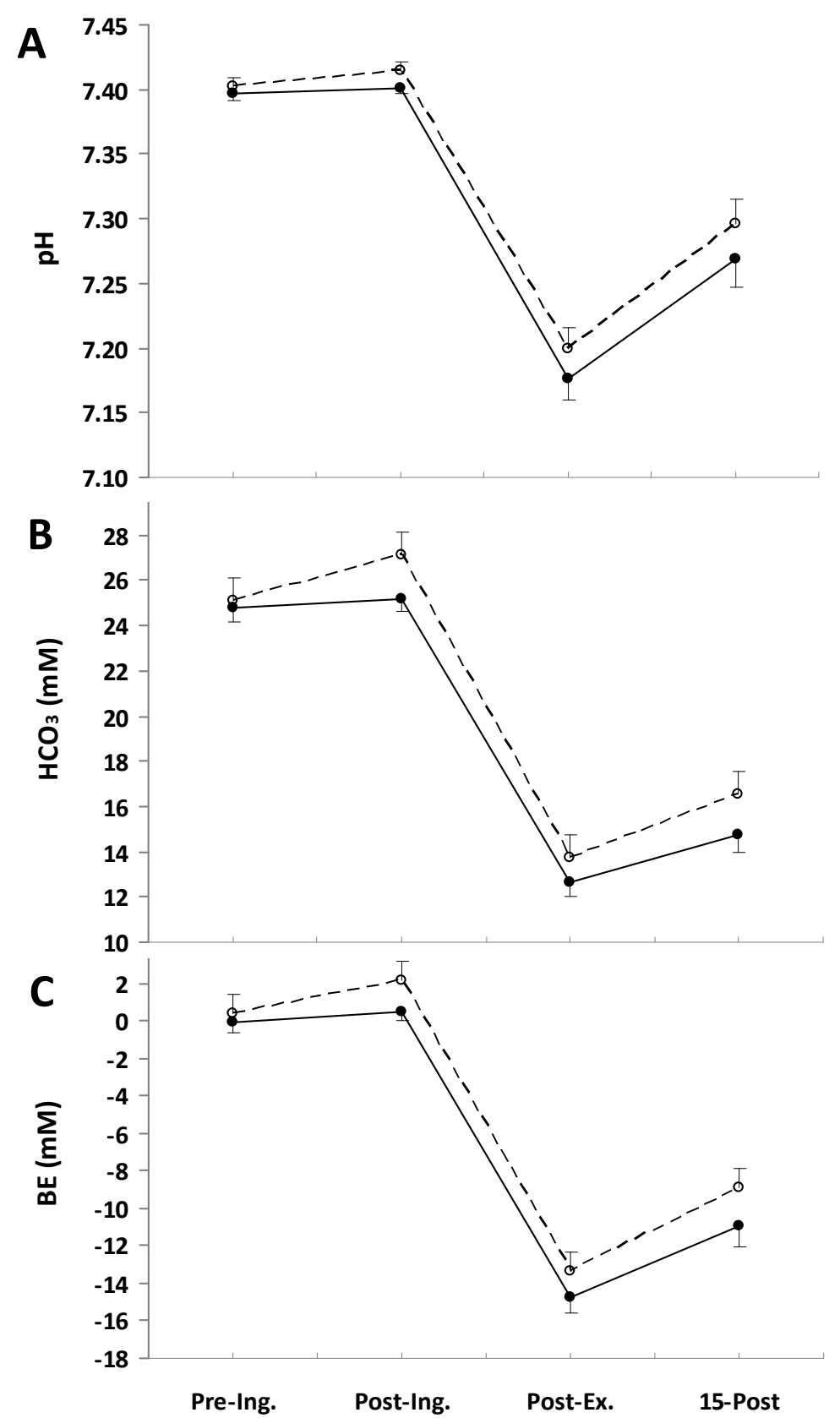

Blood LA concentration was not different after ingestion, but post-exercise was significantly higher in the $\mathrm{NaP}(12.9 \pm 0.9 \mathrm{mM})$ than in the placebo $(10.6 \pm 0.3 \mathrm{mM}, p<0.05)$ and remained elevated (nonsignificant) after 15 min of recovery (Figure 2). 
Figure 2. Blood lactate (LA) measured before placebo (•) and $\mathrm{NaP}(\mathrm{O})$ ingestion (Pre-Ing.), 60 min after ingestion (Post-Ing.), 3 min after completion of exercise (Post-Ex.), and $15 \mathrm{~min}$ of recovery (15-Post). Values are the means \pm SEM. * Significantly different from placebo at the same time point $(p<0.05)$.

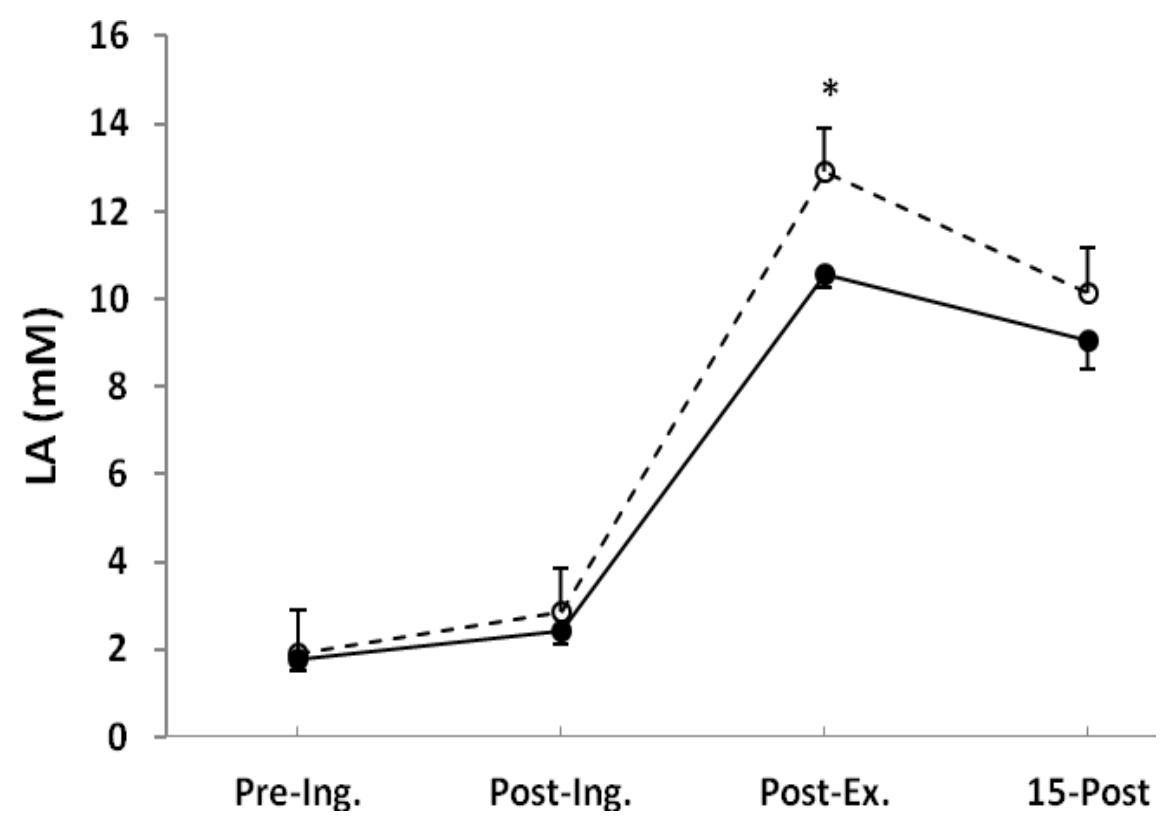

The main effect of time $(p<0.001)$ was observed in PYR, ALA and GLU concentrations in the blood (Table 1). However, there were no significant differences between the trials; NaP ingestion had no effect on measured metabolites (Table 1).

Table 1. Blood pyruvate (PYR), alanine (ALA) and glucose (GLU) concentrations at rest, after placebo or $\mathrm{NaP}$ ingestion and following exercise. Values are the means $\pm \mathrm{SEM}$.

\begin{tabular}{ccc}
\hline & Placebo & NaP \\
\hline PYR $(\mu \mathrm{M}){ }^{\#}$ & & \\
Rest & $101 \pm 18$ & $110 \pm 33$ \\
60 min after ingestion & $149 \pm 22$ & $191 \pm 51$ \\
3 min after exercise & $329 \pm 34$ & $344 \pm 34$ \\
15 min after exercise & $354 \pm 42$ & $300 \pm 69$ \\
ALA $(\mu \mathrm{M}){ }^{\#}$ & & \\
Rest & $296 \pm 21$ & $286 \pm 28$ \\
60 min after ingestion & $285 \pm 24$ & $331 \pm 23$ \\
3 min after exercise & $375 \pm 53$ & $385 \pm 34$ \\
15 min after exercise & $383 \pm 21$ & $427 \pm 45$ \\
GLU (mM) & & \\
Rest & $52.0 \pm 1.4$ & $51.7 \pm 1.4$ \\
60 min after ingestion & $51.8 \pm 0.8$ & $51.7 \pm 1.5$ \\
3 min after exercise & $63.3 \pm 2.0$ & $63.3 \pm 2.8$ \\
15 min after exercise & $58.8 \pm 1.4$ & $57.2 \pm 2.9$ \\
\hline
\end{tabular}

${ }^{\#}$ Main effect of time $(p<0.001)$. 
The NaP ingestion resulted in no alterations in the overall $\dot{\mathrm{V}} \mathrm{O}_{2}$ response during the exercise (Table 2). Consistent with this, the slow component amplitude was not significantly different between treatments. There were also no marked differences in $\dot{\mathrm{V}} \mathrm{CO}_{2}$ during the final minute of the exercise, being $4.08 \pm 0.10 \mathrm{~L} \cdot \mathrm{min}^{-1}$ in the placebo trial and $4.03 \pm 0.09 \mathrm{~L} \cdot \mathrm{min}^{-1}$ in the NaP trial; therefore, the respiratory exchange ratio (RER) was also not altered by the NaP ingestion (Table 2). Furthermore, no effects of NaP were noted in $\dot{\mathrm{V}} \mathrm{E}$ (Table 2).

Table 2. Gas exchange, ventilation and heart rate responses during and after severe-intensity exercise following placebo and sodium pyruvate ingestion. Values are the means $\pm \mathrm{SEM}$.

\begin{tabular}{ccc}
\hline & Placebo & NaP \\
\hline $\mathrm{O}_{2}$ uptake, $\mathrm{L} \mathrm{min}^{-1}$ & & \\
Baseline & $1.07 \pm 0.02$ & $1.01 \pm 0.05$ \\
End-exercise & $3.52 \pm 0.06$ & $3.44 \pm 0.06$ \\
Slow component amplitude & $0.53 \pm 0.04$ & $0.50 \pm 0.04$ \\
$\mathrm{CO}_{2}$ output, L min & -1 & \\
Baseline & & $0.78 \pm 0.04$ \\
End-exercise & $0.81 \pm 0.03$ & $4.03 \pm 0.09$ \\
Minute ventilation, $\mathrm{L} \mathrm{min}^{-1}$ & $4.08 \pm 0.10$ & $22 \pm 1$ \\
Baseline & & $116 \pm 6$ \\
End-exercise & $121 \pm 5$ & \\
Respiratory exchange ratio & & $0.77 \pm 0.02$ \\
Baseline & $0.76 \pm 0.02$ & $1.17 \pm 0.02$ \\
End-exercise & $1.16 \pm 0.02$ & $87 \pm 4$ \\
Heart rate, beats min & & \\
Baseline & $86 \pm 3$ & $171 \pm 3$ \\
End-exercise & $171 \pm 2$ & \\
\hline
\end{tabular}

\section{Discussion}

The major new finding of this study was that the single oral NaP ingestion modified the blood acid-base status. Moreover, the post-exercise blood LA concentration was higher in the NaP trial. The gas exchange and the ventilation responses were not affected by the NaP consumption.

The buffering character of PYR has been presented in earlier studies [5-7,22]. However, previously, PYR was infused intravenously at very high doses [5-7,22]. The intravenous infusion raised the arterial PYR concentration from 0.12 to $5.68 \mathrm{mM} 30 \mathrm{~min}$ after the start of the infusion. At the same time, blood $\mathrm{pH}, \mathrm{BE}$ and $\mathrm{HCO}_{3}{ }^{-}$reached 7.54, $12 \mathrm{mM}$ and $38 \mathrm{mM}$, respectively [5]. In the present study, we noted the modification in the blood acid-base status, but without any alteration in the blood PYR concentration. Similarly, Morrison et al. [11] showed that an acute oral PYR intake, even at a dose of $25 \mathrm{~g}$, did not modify PYR concentration in the whole blood or in the plasma. The inability to detect any elevation of PYR in blood, as well as the increased borborygmus and flatulence in the subjects consuming higher doses led Morrison and coworkers to suggestion that it could be decarboxylated in the stomach or eliminated through the feces; however, rapid clearance by the liver or muscles has not been ruled out [11]. Since the blood acid-base status has been ameliorated after an oral NaP ingestion, its absorption from the digestive system may occur. 
It has been postulated that the buffering PYR mechanism may be based on its transport to the cells [23]. The uptake of PYR by the cells depends on the monocarboxylate transporter system. This system is located in the plasma membrane, and it transports monocarboxylates together with $\mathrm{H}^{+}$[24]. The transfer of the hydrogen proton into the cytosol effectively raises the blood $\mathrm{pH}$. Since the intravenous PYR infusion failed to increase muscle PYR content [25], it would be expected that the majority of PYR would be cleared by the liver [11]. Considering PYR as an excellent gluconeogenic precursor, it can be either released as GLU or stored as glycogen. Although there is no significant differences in blood GLU over time after oral intake [11] or intravenous infusion [25], the trend toward the higher RER values during a low intensity exercise, suggesting a higher rate of carbohydrate oxidation after PYR supplementation, has been presented [18].

It has been also proposed that the PYR buffering properties derived from its ability to be reduced to LA. During this reaction, $\mathrm{H}^{+}$is utilized [9,23]. However, the stable blood LA over $4 \mathrm{~h}$ rest, even after ingestion of $25 \mathrm{~g}$ PYR, has been previously observed [11]. Since after a high intensity exercise, the cytoplasmic NADH/NAD ${ }^{+}$ratio in the skeletal muscle elevates [26], it seems that PYR could react with the excess of NADH, forming LA. The increased LA level in the NaP trial may indicate that ingested PYR could be partially reduced to LA. However, despite an increased LA concentration, the LA/PYR ratio was not different between the trials (not shown). Moreover, the effect of $\mathrm{NaP}$ was similar to that previously reported after sodium bicarbonate treatment; the modification of blood acid-base status and the elevation of a post-exercise LA concentration [27-30].

The results of the studies examining the net exchange of PYR across working skeletal muscle in humans reported that the muscles release PYR both during long exercise at lower intensities [31,32] and a short duration, high intensity workout [33]. In the present study, the arterio-venous difference was not determined, but capillary blood PYR concentration increased after exercise. However, no difference between the trials was noted.

Another metabolic fate of PYR in a working skeletal muscle is the conversion to ALA [2]. In the reaction catalyzed by the glutamate-pyruvate transaminase (GPT), the amino group is transferred from glutamate to PYR, resulting in ALA and $\alpha$-ketoglutarate formation. During strenuous exercise, the production of ALA by muscles markedly increases [34,35]. However, daily $25 \mathrm{~g}$ of NaP in a combination with $75 \mathrm{~g}$ of dihydroxyacetone supplementation for a week do not affect the post-exercise blood ALA concentration [36]. Similarly, in the present study, the blood ALA concentration was not affected by the NaP ingestion.

The GPT reaction in a skeletal muscle is not only essential for ALA synthesis. Due to the production of $\alpha$-ketoglutarate, it may play an important role in replenishing the TCA intermediates in the muscle mitochondria at the onset of exercise [37]. The findings of Timmons et al. [38] suggest that the provision of the oxidative substrate is a factor that limits oxidative metabolism early in exercise and that increasing the availability of the substrate early in exercise allows for increased oxidative metabolism. However, the later studies indicated that a modification in TCA intermediate concentration does not affect the oxygen consumption [39,40] nor the mitochondrial respiration [41].

The slow component of the oxygen uptake kinetics represents an increasing oxygen (and energy) cost during exercise, despite the rate of the external work remaining constant, and may be implicated in the fatigue process [42]. The interventions that reduce the $\dot{\mathrm{V}} \mathrm{O}_{2}$ slow component amplitude have been reported to improve the tolerance of severe intensity exercise $[43,44]$. However, the results 
of the pre-exercise alkalinization by oral sodium bicarbonate ingestion are equivocal. Some authors found a significant reduction of the slow component [28,45], whereas others observed no effect [46,47]. In the present study, $\mathrm{NaP}$ did not influence the slow component amplitude.

The PYR supplementation has no ergogenic effect after seven or 14 days [11,12]. The PYR doses used in these studies are about $\sim 0.1 \mathrm{~g} \cdot \mathrm{kg}^{-1} \cdot \mathrm{day}^{-1}[11,12]$. Moreover, this amount has been divided into four aliquots; $2 \mathrm{~g}$, three times a day and $1 \mathrm{~g}$ with a small snack before going to bed [11]. On the contrary, high dosages of PYR in combination with dihydroxyacetone ( $25 \mathrm{~g}$ and $75 \mathrm{~g}$ per day, respectively) consumed for one week enhance the endurance capacity [48,49], but such a supplementation protocol may be distressful to the stomach.

\section{Conclusions}

Although the results showed an improvement of the blood acid-base parameters after a single dose of $\mathrm{NaP}$ ingestion, it should be noted that the limitation of the present study was the lack of its effect on performance. However, it seems plausible that single PYR ingestion could have an ergogenic capacity, especially ingested prior to high-intensity physical efforts. Therefore, further experimental work would be necessary to clarify the mechanism of PYR action.

\section{Acknowledgments}

This work was supported by the grant, No. N RSA1 002851, from the Polish Ministry of Science and Higher Education.

\section{Author Contributions}

R.O. contributed in study design, recruitment and data collection, sample analysis, data analysis and preparation of the manuscript. S.K. and D.W. contributed in sample analysis and preparation of the manuscript. R.L. contributed in overall supervision and preparation of manuscript.

\section{Conflicts of Interest}

The authors declare no conflict of interest.

\section{References}

1. Spriet, L.L.; Howlett, R.A.; Heigenhauser, G.J. An enzymatic approach to lactate production in human skeletal muscle during exercise. Med. Sci. Sports Exerc. 2000, 32, 756-763.

2. Felig, P.; Wahren, J. Amino acid metabolism in exercising man. J. Clin. Investig. 1971, 50, 2703-2714.

3. Regitz, V.; Azumi, T.; Stephan, H.; Naujocks, S.; Schaper, W. Biochemical mechanism of infarct size reduction by pyruvate. Cardiovasc. Res. 1981, 15, 652-658.

4. Kerr, P.M.; Suleiman, M.S.; Halestrap, A.P. Reversal of permeability transition during recovery of hearts from ischemia and its enhancement by pyruvate. Am. J. Physiol. 1999, 276, H496-H502.

5. Mongan, P.D.; Fontana, J.L.; Chen, R.; Bunger, R. Intravenous pyruvate prolongs survival during hemorrhagic shock in swine. Am. J. Physiol. 1999, 277, H2253-H2263. 
6. Flaherty, D.C.; Hoxha, B.; Sun, J.; Gurji, H.; Simecka, J.W.; Mallet, R.T.; Olivencia-Yurvati, A.H. Pyruvate-fortified fluid resuscitation improves hemodynamic stability while suppressing systemic inflammation and myocardial oxidative stress after hemorrhagic shock. Mil. Med. 2010, 175, 166-172.

7. Hu, S.; Bai, X.D.; Liu, X.Q.; Wang, H.B.; Zhong, Y.X.; Fang, T.; Zhou, F.Q. Pyruvate Ringer's solution corrects lactic acidosis and prolongs survival during hemorrhagic shock in rats. J. Emerg. Med. 2013, 45, 885-893.

8. Zweier, J.L.; Jacobus, W.E. Substrate-induced alterations of high energy phosphate metabolism and contractile function in the perfused heart. J. Biol. Chem. 1987, 262, 8015-8021.

9. Robergs, R.A.; Ghiasvand, F.; Parker, D. Biochemistry of exercise-induced metabolic acidosis. Am. J. Physiol. Regul. Integr. Comp. Physiol. 2004, 287, R502-R516.

10. Karetzky, M.S.; Cain, S.M. Effect of sodium pyruvate infusion on acid-base balance and gas exchange in the dog. Am. J. Physiol. 1969, 217, 1472-1476.

11. Morrison, M.A.; Spriet, L.L.; Dyck, D.J. Pyruvate ingestion for 7 days does not improve aerobic performance in well-trained individuals. J. Appl. Physiol. 2000, 89, 549-556.

12. Ebersole, K.T.; Stout, J.R.; Eckerson, J.M.; Housh, T.J.; Evetovich, T.K.; Smith, D.B. The Effect of Pyruvate Supplementation on Critical Power. J. Strength Cond. Res. 2000, 14, 132-134.

13. Olek, R.; Luszczyk, M.; Kujach, S.; Ziemann, E.; Pieszko, M.; Pischel, I.; Laskowski, R. Gdansk University of Physical Education and Sport, Gdansk, Poland. Unpublished work, 2014.

14. Siegler, J.C.; Midgley, A.W.; Polman, R.C.; Lever, R. Effects of various sodium bicarbonate loading protocols on the time-dependent extracellular buffering profile. J. Strength Cond. Res. 2010, 24, 2551-2557.

15. Turner, M.; Page, R.; Mitchell, N.; Siegler, J. The effects of Energised Greens upon blood acid-base balance during resting conditions. J. Int. Soc. Sports Nutr. 2011, 8, doi:10.1186/1550-2783-8-14.

16. Olek, R.A.; Safranow, K.; Jakubowska, K.; Olszewska, M.; Chlubek, D.; Laskowski, R. Allopurinol intake does not modify the slow component of $\dot{\mathrm{V}} \mathrm{O}_{2}$ kinetics and oxidative stress induced by severe intensity exercise. Physiol. Res. 2012, 61, 89-96.

17. Kalman, D.; Colker, C.M.; Wilets, I.; Roufs, J.B.; Antonio, J. The effects of pyruvate supplementation on body composition in overweight individuals. Nutrition 1999, 15, 337-340.

18. Koh-Banerjee, P.K.; Ferreira, M.P.; Greenwood, M.; Bowden, R.G.; Cowan, P.N.; Almada, A.L.; Kreider, R.B. Effects of calcium pyruvate supplementation during training on body composition, exercise capacity, and metabolic responses to exercise. Nutrition 2005, 21, 312-319.

19. Bearden, S.E.; Moffatt, R.J. $\mathrm{VO}_{2}$ slow component: To model or not to model? Med. Sci. Sports Exerc. 2001, 33, 677-680.

20. Grocott, M.P.; Martin, D.S.; Levett, D.Z.; McMorrow, R.; Windsor, J.; Montgomery, H.E. Arterial blood gases and oxygen content in climbers on Mount Everest. N. Engl. J. Med. 2009, 360, 140-149.

21. Maughan, R.J. A simple, rapid method for the determination of glucose, lactate, pyruvate, alanine, 3-hydroxybutyrate and acetoacetate on a single 20-mul blood sample. Clin. Chim. Acta 1982, 122, 231-240.

22. Mongan, P.D.; Karaian, J.; van der Schuur, B.M.; Via, D.K.; Sharma, P. Pyruvate prevents poly-ADP ribose polymerase (PARP) activation, oxidative damage, and pyruvate dehydrogenase deactivation during hemorrhagic shock in swine. J. Surg. Res. 2003, 112, 180-188. 
23. Zhou, F.Q. Pyruvate in the correction of intracellular acidosis: A metabolic basis as a novel superior buffer. Am. J. Nephrol. 2005, 25, 55-63.

24. Poole, R.C.; Halestrap, A.P. Transport of lactate and other monocarboxylates across mammalian plasma membranes. Am. J. Physiol. 1993, 264, C761-C782.

25. Constantin-Teodosiu, D.; Simpson, E.J.; Greenhaff, P.L. The importance of pyruvate availability to PDC activation and anaplerosis in human skeletal muscle. Am. J. Physiol. 1999, 276, E472-E478.

26. Sahlin, K.; Katz, A.; Henriksson, J. Redox state and lactate accumulation in human skeletal muscle during dynamic exercise. Biochem. J. 1987, 245, 551-556.

27. Stephens, T.J.; McKenna, M.J.; Canny, B.J.; Snow, R.J.; McConell, G.K. Effect of sodium bicarbonate on muscle metabolism during intense endurance cycling. Med. Sci. Sports Exerc. 2002, 34, 614-621.

28. Berger, N.J.; McNaughton, L.R.; Keatley, S.; Wilkerson, D.P.; Jones, A.M. Sodium bicarbonate ingestion alters the slow but not the fast phase of VO2 kinetics. Med. Sci. Sports Exerc. 2006, 38, 1909-1917.

29. Lindh, A.M.; Peyrebrune, M.C.; Ingham, S.A.; Bailey, D.M.; Folland, J.P. Sodium bicarbonate improves swimming performance. Int. J. Sports Med. 2008, 29, 519-523.

30. Vanhatalo, A.; McNaughton, L.R.; Siegler, J.; Jones, A.M. Effect of induced alkalosis on the power-duration relationship of "all-out" exercise. Med. Sci. Sports Exerc. 2010, 42, 563-570.

31. Henderson, G.C.; Horning, M.A.; Lehman, S.L.; Wolfel, E.E.; Bergman, B.C.; Brooks, G.A. Pyruvate shuttling during rest and exercise before and after endurance training in men. J. Appl. Physiol. 2004, 97, 317-325.

32. Stellingwerff, T.; Leblanc, P.J.; Hollidge, M.G.; Heigenhauser, G.J.; Spriet, L.L. Hyperoxia decreases muscle glycogenolysis, lactate production, and lactate efflux during steady-state exercise. Am. $J$. Physiol. Endocrinol. Metab. 2006, 290, E1180-E1190.

33. Bangsbo, J.; Gibala, M.J.; Krustrup, P.; Gonzalez-Alonso, J.; Saltin, B. Enhanced pyruvate dehydrogenase activity does not affect muscle $\mathrm{O}_{2}$ uptake at onset of intense exercise in humans. Am. J. Physiol. Regul. Integr. Comp. Physiol. 2002, 282, R273-R280.

34. Babij, P.; Matthews, S.M.; Rennie, M.J. Changes in blood ammonia, lactate and amino acids in relation to workload during bicycle ergometer exercise in man. Eur. J. Appl. Physiol. Occup. Physiol. 1983, 50, 405-411.

35. Bassini, A.; Magalhaes-Neto, A.M.; Sweet, E.; Bottino, A.; Veiga, C.; Tozzi, M.B.; Pickard, M.B.; Cameron, L.C. Caffeine decreases systemic urea in elite soccer players during intermittent exercise. Med. Sci. Sports Exerc. 2013, 45, 683-690.

36. Stanko, R.T.; Diven, W.F.; Robertson, R.J.; Spina, R.J.; Galbreath, R.W.; Reilly, J.J., Jr.; Goss, F.L. Amino acid arterial concentration and muscle exchange during submaximal arm and leg exercise: The effect of dihydroxyacetone and pyruvate. J. Sports Sci. 1993, 11, 17-23.

37. Sahlin, K.; Katz, A.; Broberg, S. Tricarboxylic acid cycle intermediates in human muscle during prolonged exercise. Am. J. Physiol. 1990, 259, C834-C841.

38. Timmons, J.A.; Gustafsson, T.; Sundberg, C.J.; Jansson, E.; Greenhaff, P.L. Muscle acetyl group availability is a major determinant of oxygen deficit in humans during submaximal exercise. Am. J. Physiol. 1998, 274, E377-E380. 
39. Howlett, R.A.; Heigenhauser, G.J.; Hultman, E.; Hollidge-Horvat, M.G.; Spriet, L.L. Effects of dichloroacetate infusion on human skeletal muscle metabolism at the onset of exercise. Am. J. Physiol. 1999, 277, E18-E25.

40. Koppo, K.; Wilkerson, D.P.; Bouckaert, J.; Wilmshurst, S.; Campbell, I.T.; Jones, A.M. Influence of DCA on pulmonary $\dot{\mathrm{V}} \mathrm{O} 2$ kinetics during moderate-intensity cycle exercise. Med. Sci. Sports Exerc. 2004, 36, 1159-1164.

41. Bangsbo, J.; Gibala, M.J.; Howarth, K.R.; Krustrup, P. Tricarboxylic acid cycle intermediates accumulate at the onset of intense exercise in man but are not essential for the increase in muscle oxygen uptake. Pflugers Arch. 2006, 452, 737-743.

42. Zoladz, J.A.; Korzeniewski, B. Physiological background of the change point in $\mathrm{VO}_{2}$ and the slow component of oxygen uptake kinetics. J. Physiol. Pharmacol. 2001, 52, 167-184.

43. Bailey, S.J.; Winyard, P.G.; Vanhatalo, A.; Blackwell, J.R.; Dimenna, F.J.; Wilkerson, D.P.; Jones, A.M. Acute L-arginine supplementation reduces the $\mathrm{O}_{2}$ cost of moderate-intensity exercise and enhances high-intensity exercise tolerance. J. Appl. Physiol. 2010, 109, 1394-1403.

44. Lansley, K.E.; Winyard, P.G.; Bailey, S.J.; Vanhatalo, A.; Wilkerson, D.P.; Blackwell, J.R.; Gilchrist, M.; Benjamin, N.; Jones, A.M. Acute dietary nitrate supplementation improves cycling time trial performance. Med. Sci. Sports Exerc. 2011, 43, 1125-1131.

45. Kolkhorst, F.W.; Rezende, R.S.; Levy, S.S.; Buono, M.J. Effects of sodium bicarbonate on $\mathrm{VO}_{2}$ kinetics during heavy exercise. Med. Sci. Sports Exerc. 2004, 36, 1895-1899.

46. Santalla, A.; Perez, M.; Montilla, M.; Vicente, L.; Davison, R.; Earnest, C.; Lucia, A. Sodium bicarbonate ingestion does not alter the slow component of oxygen uptake kinetics in professional cyclists. J. Sports Sci. 2003, 21, 39-47.

47. Zoladz, J.A.; Duda, K.; Majerczak, J.; Domanski, J.; Emmerich, J. Metabolic alkalosis induced by pre-exercise ingestion of $\mathrm{NaHCO}_{3}$ does not modulate the slow component of $\mathrm{VO}_{2}$ kinetics in humans. J. Physiol. Pharmacol. 1997, 48, 211-223.

48. Stanko, R.T.; Robertson, R.J.; Galbreath, R.W.; Reilly, J.J., Jr.; Greenawalt, K.D.; Goss, F.L. Enhanced leg exercise endurance with a high-carbohydrate diet and dihydroxyacetone and pyruvate. J. Appl. Physiol. 1990, 69, 1651-1656.

49. Stanko, R.T.; Robertson, R.J.; Spina, R.J.; Reilly, J.J., Jr.; Greenawalt, K.D.; Goss, F.L. Enhancement of arm exercise endurance capacity with dihydroxyacetone and pyruvate. J. Appl. Physiol. 1990, 68, 119-124.

(C) 2014 by the authors; licensee MDPI, Basel, Switzerland. This article is an open access article distributed under the terms and conditions of the Creative Commons Attribution license (http://creativecommons.org/licenses/by/3.0/). 